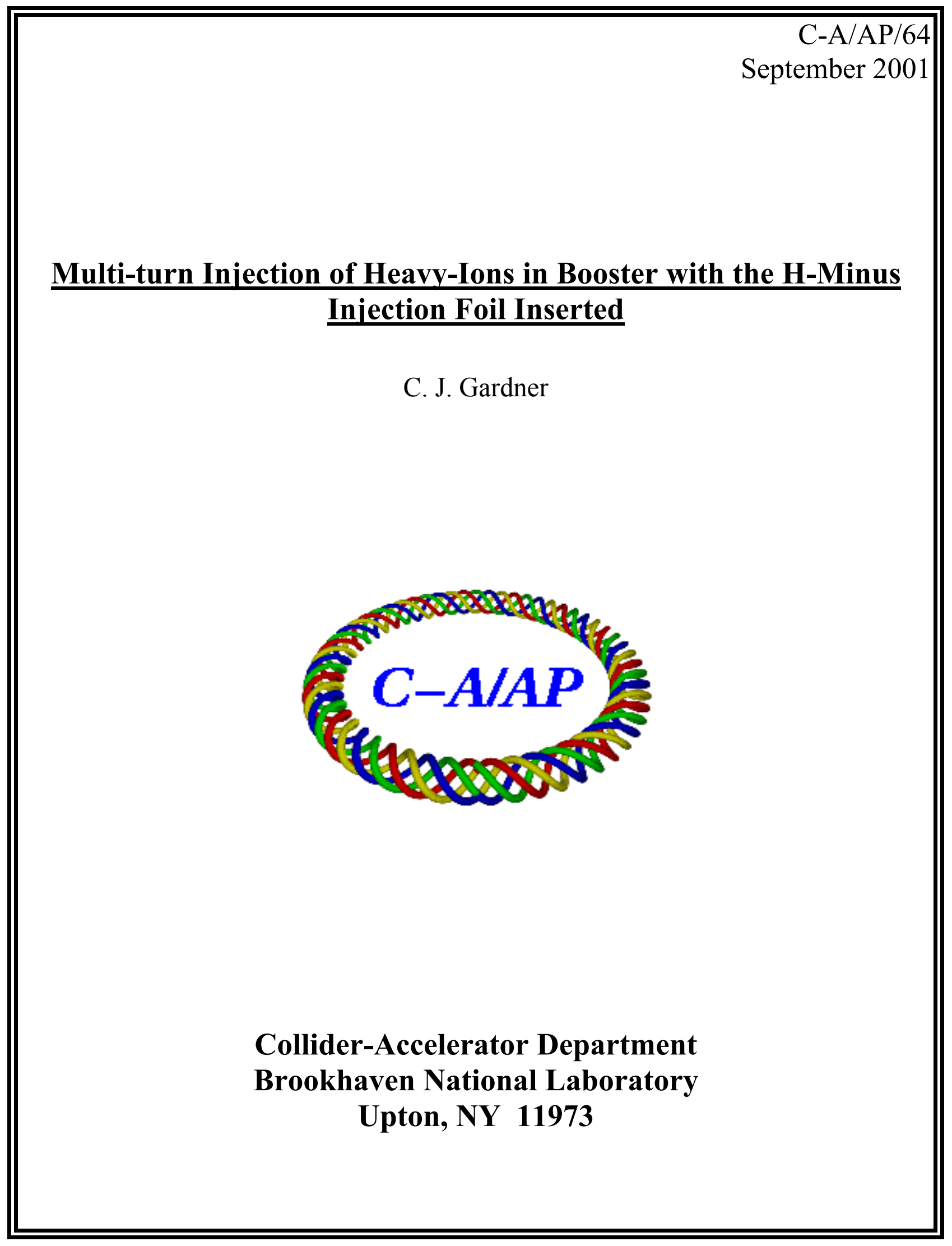




\title{
Multi-turn Injection of Heavy-Ions in Booster with the H-Minus Injection Foil Inserted
}

\author{
C.J. Gardner
}

September 14, 2001

When the Booster Application Facility (BAF) becomes operational, we will want to inject and accelerate heavy ion and proton beams in Booster on a ppm (pulse-to-pulse modulation) basis. The heavy ions will be delivered to BAF for biology experiments while protons will be delivered to the experimental hall for high energy physics. One item that potentially stands in the way of ppm operation is the H-minus injection foil. Normally the foil is inserted into the Booster aperture for proton operation and retracted during heavy ion operation. The motorized device that does this can not move the foil in and out fast enough for ppm operation. (Even if it could, one would worry about the lifetime of such a device.) In this note we show that in principle the injection foil can be left in its nominal position for H-minus injection without impinging on the available aperture for the injection and acceleration of heavy ions.

\section{The Injection Foils}

Five foils are available for H-minus injection. They are mounted on a flat rotatable circular frame that has six equally spaced slots, five of which hold a foil and one which is left open (blank). The plane of the frame is perpendicular to the beam line and is located $0.283 \mathrm{~m}$ downstream of dipole DHC5 and $0.712 \mathrm{~m}$ upstream of quadrupole QHC6. Each foil is inserted from the radially inward side of the beam pipe by rotating its slot into the Booster aperture. The five foils are identical except for the position of the inserted foil edge with respect to the beam pipe centerline. These positions are listed in Table 1. (Here the minus signs indicate that the foil edges are on the radially inward side of the beam pipe centerline.) The foil material is carbon with a surface density of $200 \mu \mathrm{g} / \mathrm{cm}^{2}$. The 
nominal foil for proton operation is the one in slot 3 ; its edge is 1 inch to the inside of the beam pipe centerline.

Table 1: H-Minus Injection Foils

\begin{tabular}{|c|c|c|}
\hline Slot & Foil & Foil Edge \\
\hline 1 & Blank & (Inches) \\
2 & Carbon & -0.75 \\
3 & Carbon & -1.00 \\
4 & Carbon & -1.00 \\
5 & Carbon & -1.125 \\
6 & Carbon & -1.125 \\
\hline
\end{tabular}

\section{Clearing the Foil at Injection}

With the foil retracted, the horizontal limiting aperture in Booster is the F6 septum magnet and the horizontal acceptance is $184 \pi \mathrm{mm}$ milliradians. This is computed using the MAD code with the Booster tunes set to 4.76 . (These are the typical tunes during heavy ion injection.) The resulting acceptance envelope in the region of the foil is shown in Figure 1. Here the red curves are the envelope; the vertical green line indicates the aperture at the downstream end of the C3 inflector.

When inserted without any local orbit bumps, the foil becomes the limiting aperture of the machine. With the foil edge 1 inch to the inside of the beam pipe centerline, the horizontal acceptance is reduced to $60 \pi \mathrm{mm}$ milliradians. The resulting acceptance envelope is shown in Figure 2 where the inserted foil is indicated by the vertical blue line.

\subsection{The C6 Three-Bump}

In order to increase the available aperture with the foil inserted, the closed orbit must be bumped away from the foil. This can be done with a local three-bump produced by horizontal dipole correctors DHCC4, DHCC6 and DHCC8. The bump is centered on corrector DHCC6 and is called the C6 three-bump. (DHCC6 is $0.287 \mathrm{~m}$ downstream of the foil.) With the correctors adjusted so that the position of the bumped orbit at the foil is 
$21 \mathrm{~mm}$ to the outside of the beam pipe centerline, the F6 septum magnet again becomes the limiting aperture and the horizontal acceptance is again $184 \pi \mathrm{mm}$ milliradians. The three-bump and acceptance envelope are shown in Figure 3. Here the black curve is the three-bump and the green and blue vertical lines indicate the apertures at the C3 inflector and foil respectively. Note that the envelope comes close to the inflector and foil apertures and nearly reaches the $75 \mathrm{~mm}$ aperture in the QHC6 quadrupole; there is not much additional room for the beam at these locations. The angular kicks required in the three correctors are 2.02, -1.36 , and 1.99 milliradians respectively. (Here a positive kick is in the radially outward direction.) The integrated strength of each correction dipole is $0.975 \times 10^{-4} \mathrm{Tm} / \mathrm{A}$ as reported by Thern [1], so the currents required in the three dipoles for a beam with 1 Tm rigidity are 20.7, -13.9 , and 20.4 Amps respectively.

During heavy ion injection, the C3 injection bump (produced by fast dipole kickers KDHC1, KDHC3, KDHC7 and KDHD1) initially places the closed orbit near the septum at the downstream end of the inflector. This bump is the black curve shown in Figure 4. With the C6 three-bump adjusted so that the circulating beam clears the foil (as in Figure 3), one then obtains the superposition of bumps indicated by the blue curve. As the injection bump is collapsed away from the septum, the injected beam

is contained in the envelopes indicated by the red curves in Figures 5, 6, 7 and 8 . The positions of the bumped orbit at the end of the inflector in the figures are respectively 37.5, 24, 10 and $0 \mathrm{~mm}$ with respect to the beam pipe centerline. (The septum is $47.5 \mathrm{~mm}$ from the centerline.) Here one sees that all of the injected beam clears the foil as the bump collapses. With the injection bump completely collapsed, one is left with only the C6 three-bump as in Figure 3.

\subsection{The Slow Injection Bump}

The so-called "slow injection bump" may also be used to bump the orbit away from the foil. This bump is normally used to center the orbit on the available aperture at the foil during proton operation. It is produced by the flat trim windings on dipoles DHC4, DHC8, and DHD1. (The various windings on the Booster dipoles are described in Reference [2]. The flat trims on DHC4, DHC8, and DHD1 are connected to 50-Amp power supplies.) With the currents in the flat trims adjusted so that the position of the bumped orbit at the foil is $21 \mathrm{~mm}$ to the outside of the beam pipe 
centerline, the F6 septum magnet is the limiting aperture and the horizontal acceptance is $184 \pi \mathrm{mm}$ milliradians. Figure 9 shows the bump and the $184 \pi$ acceptance envelope. Here, as before, the black curve is the bump and the green and blue vertical lines indicate the apertures at the inflector and foil. The currents required in the flat trims are such that the magnetic fields in the DHC4, DHC8 and DHD1 dipoles are decreased by $1.5 \%, 0.075 \%$, and $1.65 \%$ respectively.

\section{Clearing the Foil during Acceleration}

As the beam is adiabatically accelerated after injection, the horizontal emittance becomes

$$
\pi \epsilon=\pi \epsilon_{0} p_{0} / p
$$

where $p$ is the momentum of the accelerated beam, and $p_{0}$ and $\pi \epsilon_{0}$ are the momentum and emittance at injection. We assume that the beam completely fills the horizontal acceptance at injection, so $\pi \epsilon_{0}=184 \pi \mathrm{mm}$ milliradians. The position of the bumped orbit required for the accelerated beam to clear the foil is then

$$
X=X_{0}-\left\{\sqrt{\epsilon_{0} \beta}-\sqrt{\epsilon \beta}\right\}=X_{0}-\sqrt{\epsilon_{0} \beta}\left\{1-\sqrt{p_{0} / p}\right\}
$$

where $X_{0}=21 \mathrm{~mm}$ is the position required at injection and $\beta=10.82 \mathrm{~m}$ is the lattice beta at the foil. In each of the three-bump dipoles, the current required to give bumped orbit position $X$ is

$$
I=I_{0} \frac{X}{X_{0}} \frac{p}{p_{0}}
$$

where $I_{0}$ is the current required to give position $X_{0}$ at injection momentum $p_{0}$. Defining

$$
R=\sqrt{p / p_{0}}, \quad A=\sqrt{\epsilon_{0} \beta}
$$

we then have

$$
\begin{gathered}
I=I_{0} \frac{X}{X_{0}} R^{2}, \quad X=X_{0}-A+\frac{A}{R} \\
I=\frac{I_{0}}{X_{0}}\left\{X_{0} R^{2}-A R^{2}+A R\right\}
\end{gathered}
$$


and

$$
\frac{d I}{d R}=\frac{I_{0}}{X_{0}}\left\{2 R X_{0}-2 R A+A\right\} .
$$

Thus we see that if $R \geq 1$ and

$$
X_{0}<A / 2
$$

then

$$
2 R\left(X_{0}-A\right)+A<A(1-R) \leq 0
$$

and derivative $d I / d R$ will be negative for all $p>p_{0}$. The required current will then decrease as the beam is accelerated. Putting in numbers $\epsilon_{0}=184$ mm milliradians and $\beta=10.82 \mathrm{~m}$ we find $A=44.6 \mathrm{~mm}$. The condition $X_{0}<A / 2$ is therefore satisfied for $X_{0}=21 \mathrm{~mm}$, and the currents required in the three-bump dipoles (or in the flat trim windings for the slow injection bump) will decrease as the beam is accelerated.

\section{References}

[1] R. Thern, "Booster Ring Correction Magnets", Booster Technical Note No. 224, May 20, 1994.

[2] C.J. Gardner, "The New Booster Dump and Dump Bumps", Collider-Accelerator Dept. Note C-A/AP/46, March, 2001. 


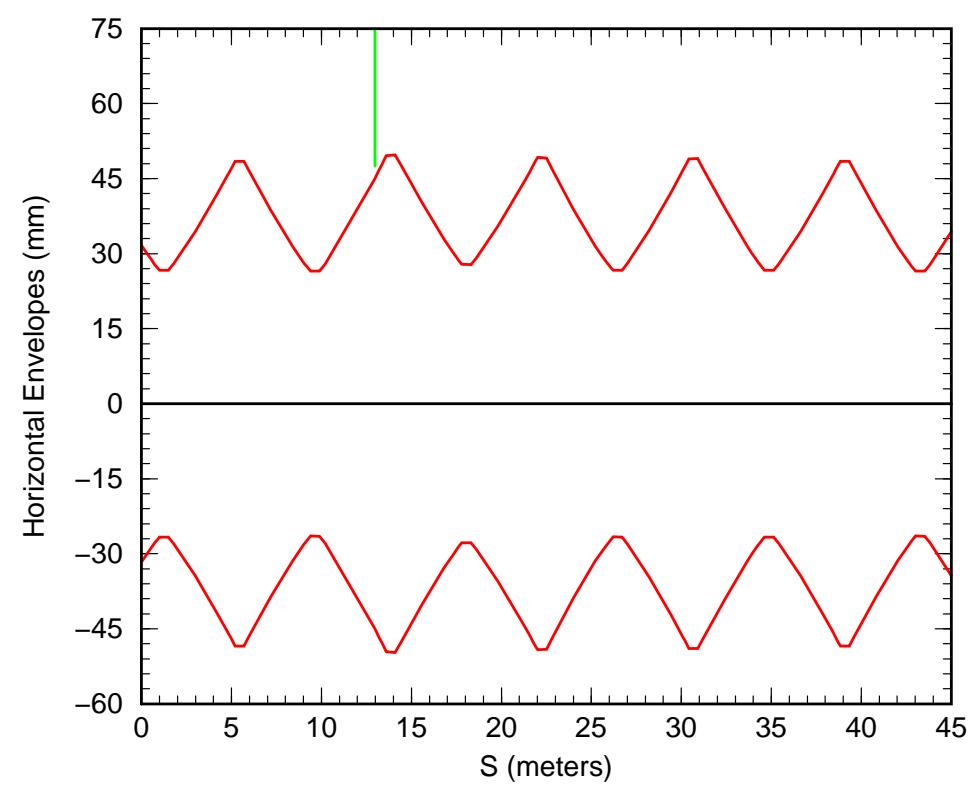

Figure 1: Acceptance Envelope with Foil Retracted

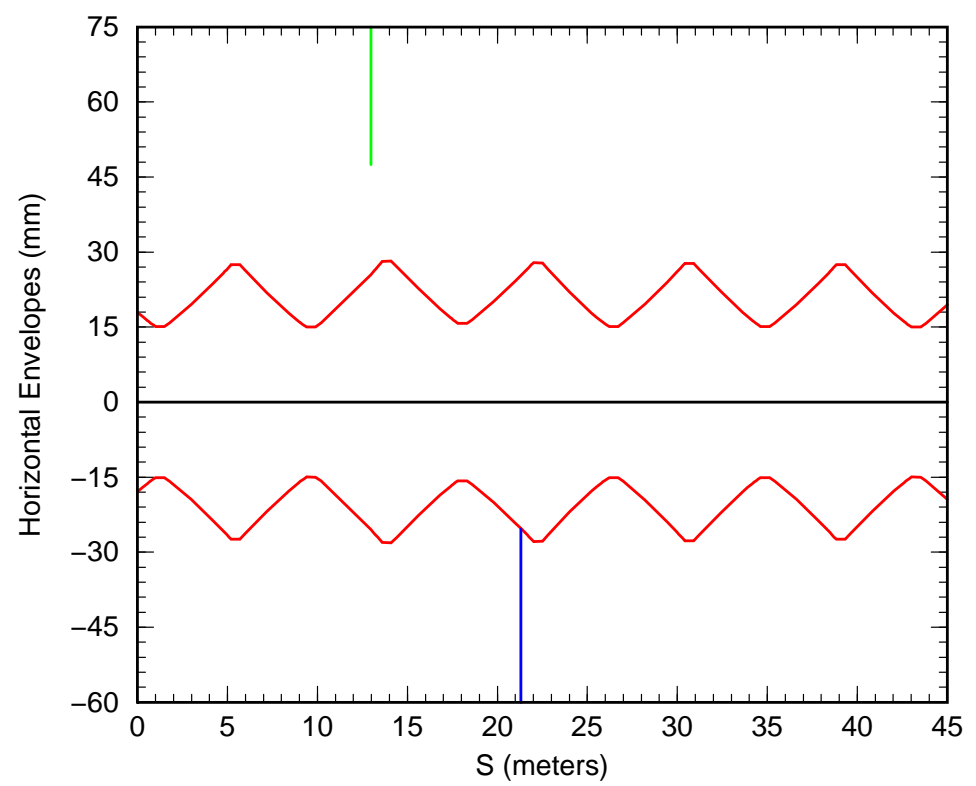

Figure 2: Acceptance Envelope with Foil Inserted 


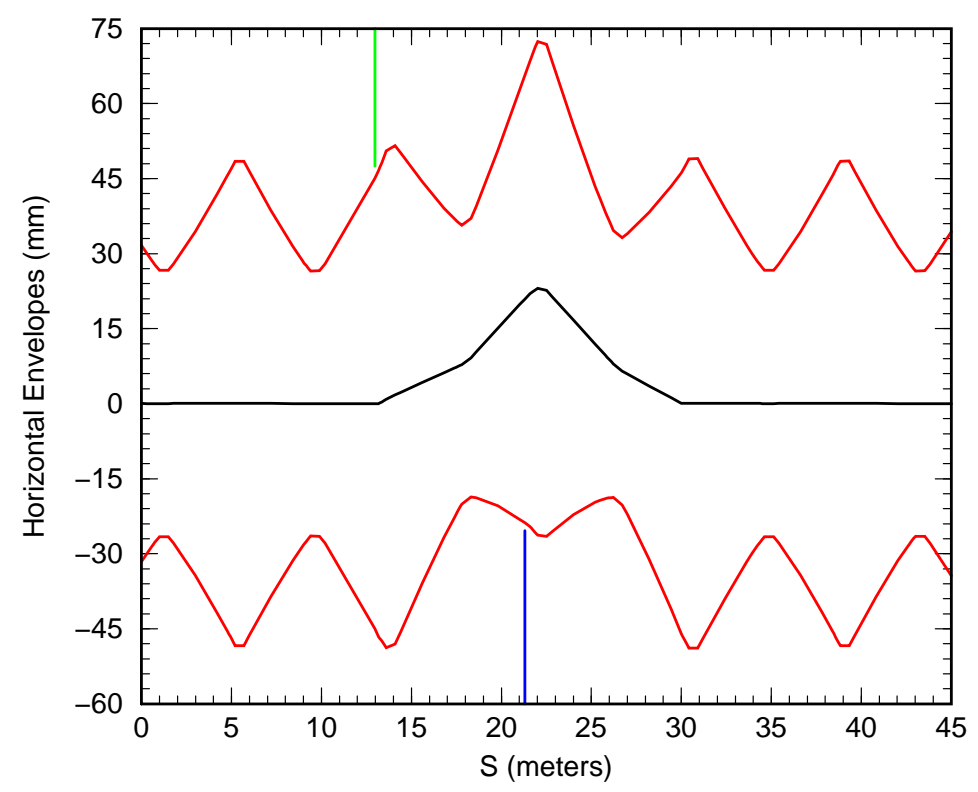

Figure 3: Acceptance Envelope with C6 Three-Bump

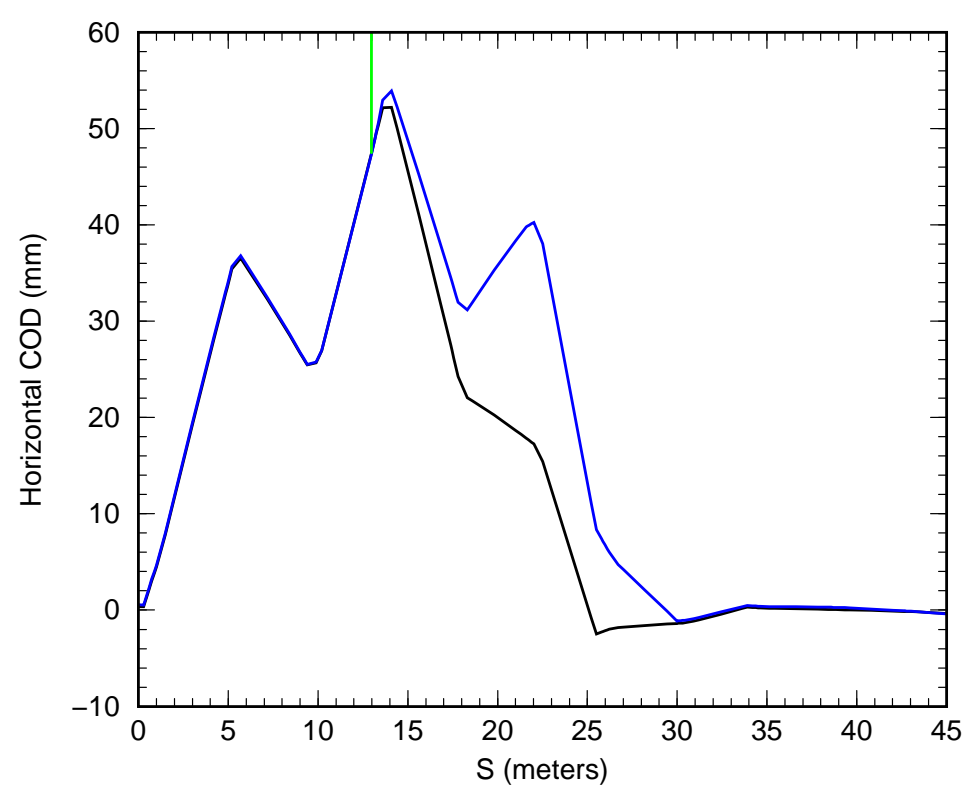

Figure 4: C3 Injection Bump and C6 Three-Bump 


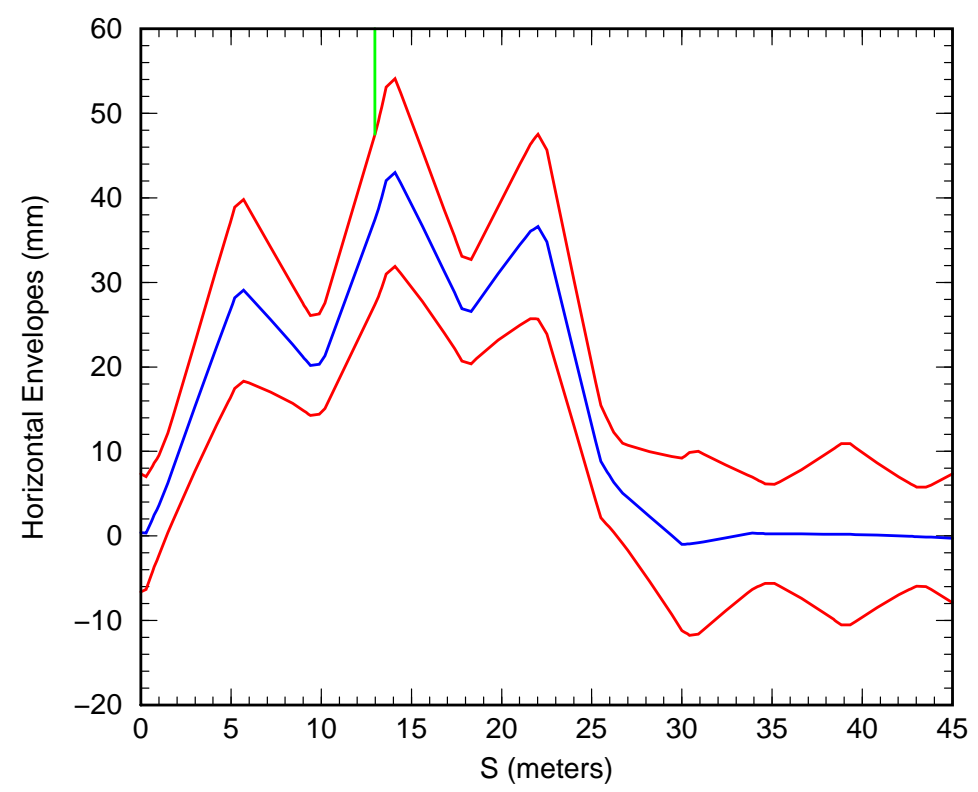

Figure 5: $37.5 \mathrm{~mm}$ Injection Bump + Three-Bump with Envelope

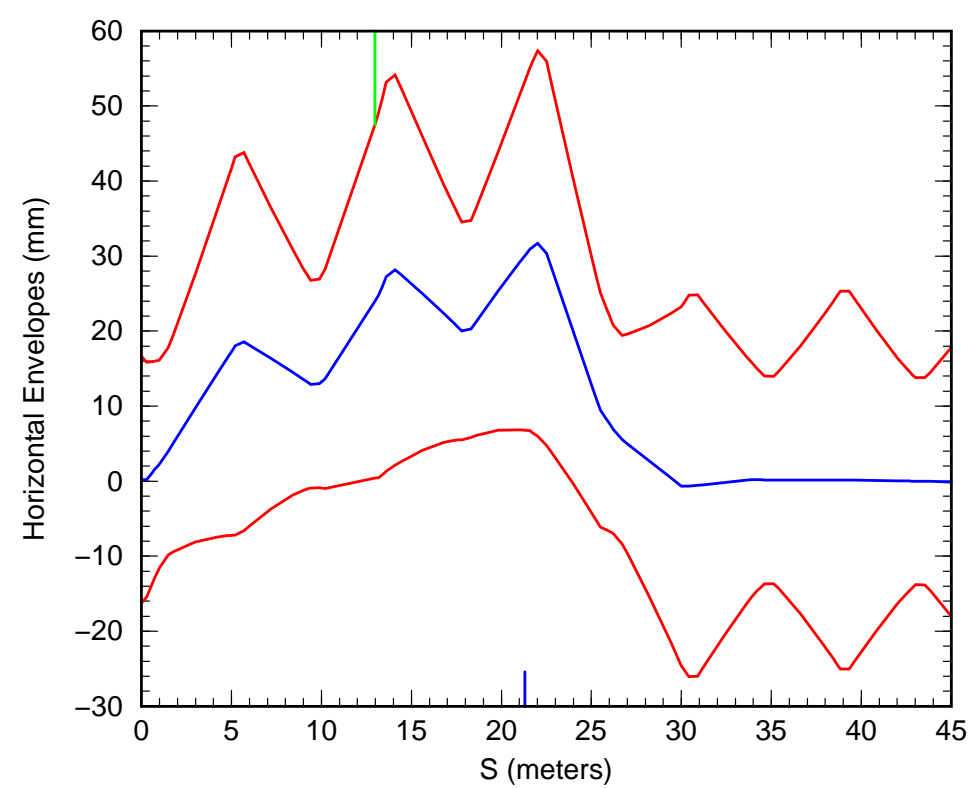

Figure 6: $24.0 \mathrm{~mm}$ Injection Bump + Three-Bump with Envelope 


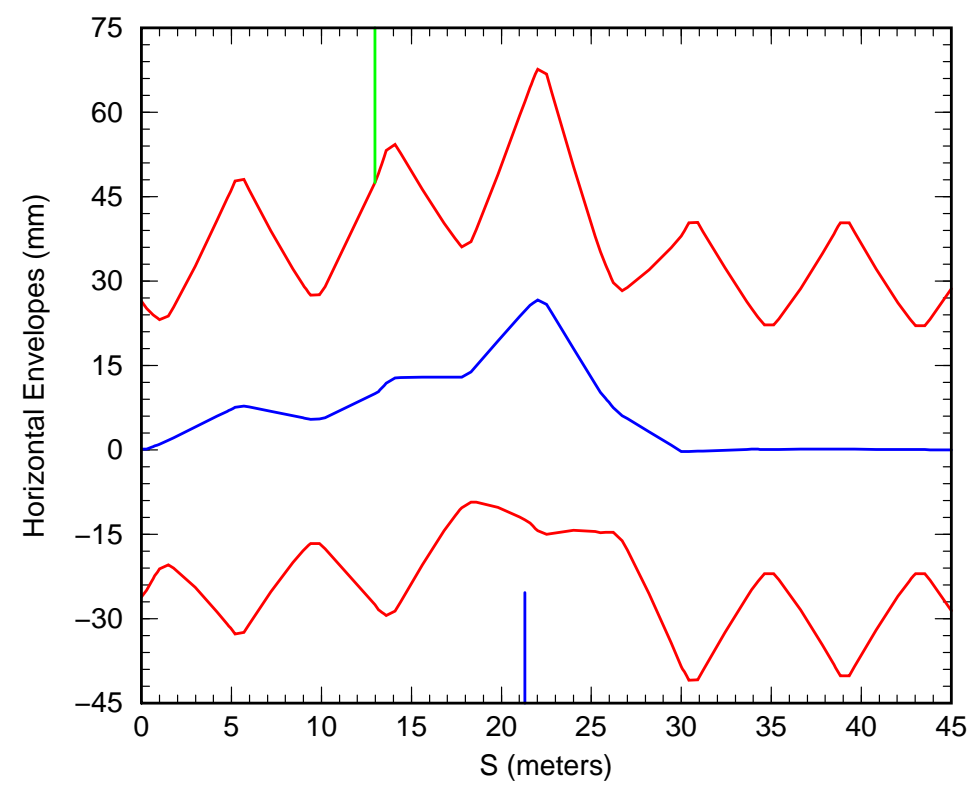

Figure 7: 10.0 mm Injection Bump + Three-Bump with Envelope

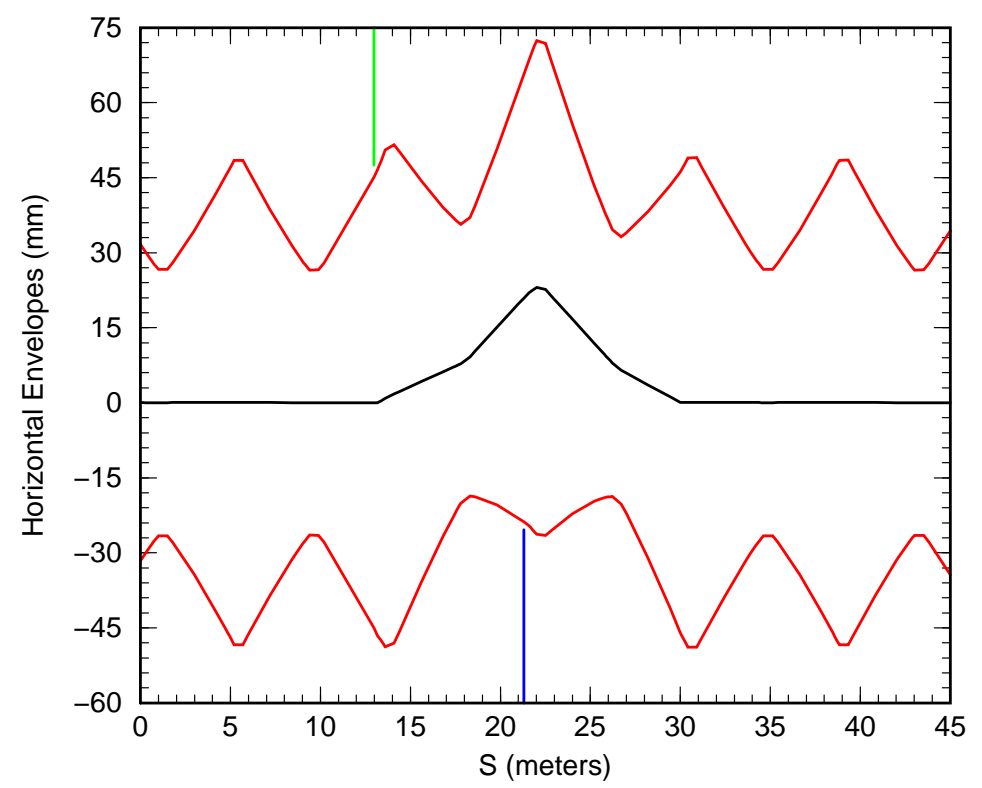

Figure 8: Three-Bump (no injection bump) with Envelope 


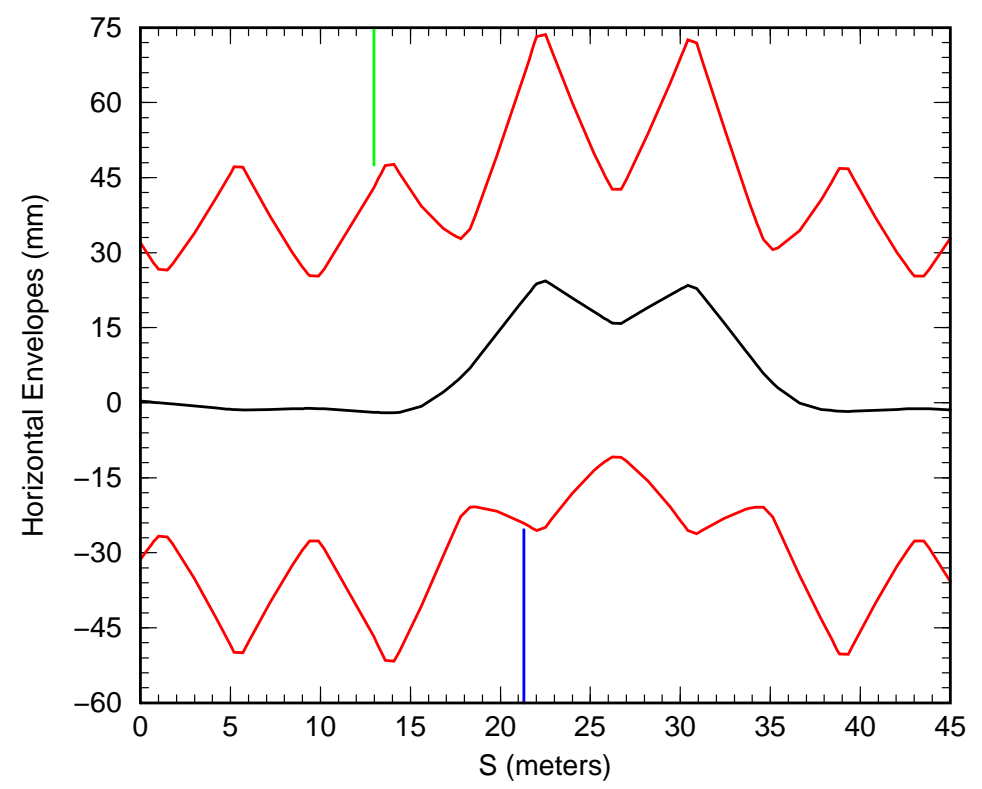

Figure 9: "Slow Injection Bump" with Envelope 